\title{
THE RADIUS OF STARLIKENESS FOR CONVEX FUNCTIONS OF COMPLEX ORDER
}

\author{
YAŞAR POLATOG̃LU, METIN BOLCAL, and ARZU ŞEN
}

Received 4 November 2003

\begin{abstract}
We will give the relation between the class of Janowski starlike functions of complex order and the class of Janowski convex functions of complex order. As a corollary of this relation, we obtain the radius of starlikeness for the class of Janowski convex functions of complex order.
\end{abstract}

2000 Mathematics Subject Classification: 30C45.

1. Introduction. Let $F$ be the class of analytic functions in $D=\{z|| z \mid<1\}$, and let $S$ denote those functions in $F$ that are univalent and normalized by $f(0)=0, f^{\prime}(0)=1$. Furthermore, let $\Omega$ be the family of functions $\omega(z)$ regular in $D$ and satisfying $\omega(0)=0$, $|\omega(z)|<1$ for $z \in D$.

For arbitrary fixed numbers $-1 \leq B<A \leq 1$, denoted by $P(A, B)$, the family of functions

$$
p(z)=1+p_{1} z+p_{2} z^{2}+\cdots,
$$

which is regular in $D$ on the condition such that

$$
p(z)=\frac{1+A \omega(z)}{1+B \omega(z)}
$$

for some functions $\omega(z) \in \Omega$ and every $z \in D$. This class was introduced by Janowski [7].

Moreover, let $S^{*}(A, B, b)$ be denoted by the family of functions $f(z) \in S$ such that $f(z)$ is in $S^{*}(A, B, b)$ if and only if $f(z) / z \neq 0$,

$$
1+\frac{1}{b}\left(z \cdot \frac{f^{\prime}(z)}{f(z)}-1\right)=p(z), \quad(b \neq 0, \text { complex })
$$

for some functions $p(z) \in P(A, B)$ and all $z$ in $D$.

Finally, let $C(A, B, b)$ denote the family of functions which are regular:

$$
1+\frac{1}{b} \cdot z \cdot \frac{f^{\prime \prime}(z)}{f^{\prime}(z)}=p(z), \quad(b \neq 0, \text { complex })
$$

for some functions $p(z) \in P(A, B)$ and every $z$ in $D$. 
We note that $P(-1,1)$ is the class of Caratheodory functions, and therefore the class $C(A, B, b)$ contains the following classes. $b=1, C(1,-1,1)=C$ is the well-known class of convex functions [2], and $C(1,-1, b)=C(b)$ is the class of convex functions of complex order $[7,8] . C(1,-1,1-\beta),(0 \leq \beta<1)$ is the class of convex functions of order $\beta$ [9]. For $A=1, B=-1, b=e^{-i \lambda} \cdot \cos \lambda,|\lambda|<\pi / 2$ is the class of functions for which $z f^{\prime}(z)$ is $\lambda$-spirallike $[3,6,11,12,13,14]$. For $A=1, B=-1, b=(1-\beta) e^{-i \lambda} \cdot \cos \lambda$, $0 \leq \beta<1,|\lambda|<\pi / 2$ is the class of functions for which $z f^{\prime}(z)$ is $\lambda$-spirallike of order $\beta$ $[3,6,11,12,13,14]$.

2. Representation theorem for the class $S^{*}(A, B, b)$. The following lemma, well known as Jack's lemma, is required in our investigation.

LEMMA 2.1 $[4,5]$. Let $w(z)$ be a nonconstant and analytic function in the unit disc $D$ with $w(0)=0$. If $|w(z)|$ attains its maximum value on the circle $|z|=r$ at the point $z_{0}$, then $z_{0} w^{\prime}\left(z_{0}\right)=k w\left(z_{0}\right)$ and $k \geq 1$.

LEMMA 2.2. $e^{-i \alpha} f\left(e^{i \alpha} z\right), \alpha \in[0,2 \pi)$ is in $C(A, B, b)$ whenever $f(z)$ is in $C(A, B, b)$.

Proof. If $f(z) \in C(A, B, b)$, then

$$
g(z)=e^{-i \alpha} f\left(e^{i \alpha} z\right) \Longrightarrow 1+\frac{1}{b} z \frac{g^{\prime}(z)}{g(z)}=1+\frac{1}{b}\left(e^{i \alpha} z\right) \frac{f^{\prime}\left(e^{i \alpha} z\right)}{f\left(e^{i \alpha} z\right)}
$$

We note that similarly the class $S^{*}(A, B, b)$ is invariant under the rotation so that $e^{-i \alpha} f\left(e^{i \alpha} z\right), \alpha \in[0,2 \pi)$ is in $S^{*}(A, B, b)$ whenever $f(z)$ is in $S^{*}(A, B, b)$.

LEMMA 2.3. If $g(z) \in S^{*}(A, B, b)$, then

$$
g(z)= \begin{cases}z(1+B w(z))^{b(A-B) / B}, & B \neq 0, k=1 \\ z e^{b A w(z)}, & B=0, k=1\end{cases}
$$

for some $w(z) \in \Omega$ and for all $z$ in $D$, and conversely.

Proof. The proof of this lemma is completed in four steps, and we have used Nicola Tuneski's technique for the special case of $k=1$ [15].

FIRST STEP. If $B \neq 0$ and

$$
g(z)=z(1+B w(z))^{b(A-B) / B},
$$

then by taking logarithmic derivative of (2.3) followed by a brief computation using Jack's lemma and the definition of subordination, we obtain

$$
1+\frac{1}{b}\left(z \frac{g^{\prime}(z)}{g(z)}-1\right)=\frac{1+A w(z)}{1+B w(z)}, \text { for } k=1
$$

and so from the definition of $S^{*}(A, B, b)$ it follows that $g(z) \in S^{*}(A, B, b)$. (See [10].) 
SECOND STEP. If $B=0$, then we have $g(z)=z e^{b A w(z)}$. Similarly, we get

$$
1+\frac{1}{b}\left(z \frac{g^{\prime}(z)}{g(z)}-1\right)=1+A w(z), \text { for } k=1 .
$$

The equality shows that $g(z) \in S^{*}(A, B, b)$.

THIRD STEP. Conversely, if $g(z) \in S^{*}(A, B, b)$ and $B \neq 0$, then we have

$$
1+\frac{1}{b}\left(z \frac{g^{\prime}(z)}{g(z)}-1\right)=\frac{1+A w(z)}{1+B w(z)}
$$

Equation (2.6) can be written in the form

$$
\frac{g^{\prime}(z)}{g(z)}=\frac{b(A-B)(w(z) / z)}{1+B w(z)}+\frac{1}{z}
$$

If we use Jack's lemma in (2.7) for $k=1$, we obtain

$$
\frac{g^{\prime}(z)}{g(z)}=\frac{b(A-B) w^{\prime}(z)}{1+B w(z)}+\frac{1}{z}
$$

Integrating both sides of equality (2.8), we get (2.3).

FOURTH STEP. Again, conversely, if $g(z) \in S^{*}(A, B, b)$ and $B=0$, then in the same way we obtain $g(z)=z e^{b A w(z)}$ which completes the proof.

LEMMA 2.4. Let $f(z)$ be regular and analytic in $D$, and normalized so that $f(0)=0$, $f^{\prime}(0)=1$. A necessary and sufficient condition for $f(z) \in C(A, B, b)$ is that for each member $g(z)=z+b_{1} z+b_{2} z^{2}+\cdots$ of $S^{*}(A, B, b)$ the following equation holds:

$$
g(z, \zeta)=z\left(\frac{f(z)-f(\zeta)}{z-\zeta}\right)^{2}, \quad \zeta, z \in D, \zeta \neq z, \zeta=n z,|n| \leq 1
$$

Proof. If $f(z) \in C(A, B, b)$, then this function is analytic, regular, and continuous in the unit disc. Therefore, equality (2.9) can be written in the form

$$
g(z)=z\left(f^{\prime}(z)\right)^{2}
$$

If we take the logarithmic derivative of equality (2.10) followed by simple calculations, we get

$$
1+\frac{1}{2 b}\left(z \frac{g^{\prime}(z)}{g(z)}-1\right)=1+\frac{1}{b} z \frac{f^{\prime \prime}(z)}{f^{\prime}(z)}=\frac{1+A w(z)}{1+B w(z)}
$$

On the other hand, $b$ is a complex number and $b \neq 0$. Therefore, $b_{1}=2 b$ is a complex number and $2 b \neq 0$, thus (2.11) can be written in the form

$$
1+\frac{1}{b_{1}}\left(z \frac{g^{\prime}(z)}{g(z)}-1\right)=1+\frac{1}{b} z \frac{f^{\prime \prime}(z)}{f^{\prime}(z)} .
$$


Considering equality (2.12), the definition of $C(A, B, b)$, and the definition of $S^{*}(A, B, b)$, we obtain $g(z) \in S^{*}(A, B, 2 b)$.

Conversely, if $g(z) \in S^{*}(A, B, b)$, and $g(z)=z((f(z)-f(\zeta)) /(z-\zeta))$ holds, then from Lemma 2.3 we get

$$
g(z)=z\left(\frac{f(z)-f(\zeta)}{z-\zeta}\right)^{2}= \begin{cases}z(1+B w(z))^{b(A-B) / B}, & B \neq 0 \\ z e^{b A w(z)}, & B=0 .\end{cases}
$$

If we take the logarithmic derivative with respect to $z$ of (2.13) followed by simple calculations, we get

$$
\begin{aligned}
1+\frac{1}{b}\left(z \frac{g^{\prime}(z)}{g(z)}-1\right) & =\frac{1}{b}\left[\frac{2 z f^{\prime}(z)}{f(z)-f(\zeta)}-\frac{z+\zeta}{z-\zeta}\right]+1-\frac{1}{b} \\
& =\frac{1+A w(z)}{1+B w(z)}, \quad B \neq 0, \\
1+\frac{1}{b}\left(z \frac{g^{\prime}(z)}{g(z)}-1\right) & =\frac{1}{b}\left[\frac{2 z f^{\prime}(z)}{f(z)-f(\zeta)}-\frac{z+\zeta}{z-\zeta}\right]+1-\frac{1}{b} \\
& =1+A w(z), \quad B=0 .
\end{aligned}
$$

Furthermore, if we write $F(z, \zeta)=(1 / b)\left[2 z f^{\prime}(z) /(f(z)-f(\zeta))-(z+\zeta) /(z-\zeta)\right]+$ $1-1 / b$, then we have

$$
\lim _{\zeta \rightarrow z} F(z, \zeta)=1+\frac{1}{b} z \frac{f^{\prime \prime}(z)}{f^{\prime}(z)}
$$

Considering relations (2.14) and (2.15) together, we obtain $f(z) \in C(A, B, b)$.

COROLlary 2.5. If $f(z) \in C(A, B, b)$, then

$$
2\left[1+\frac{1}{b}\left(z \frac{f^{\prime}(z)}{f(z)}-1\right)\right]-1=p(z)=\frac{1+A w(z)}{1+B w(z)}
$$

Proof. If we take $\zeta=0$ in $F(z, \zeta)$, we obtain the desired result of this corollary.

\section{The radius of starlikeness for the class $C(A, B, b)$}

LEMMA 3.1. If $f(z) \in C(A, B, b)$, then

$$
\left|z \frac{f^{\prime}(z)}{f(z)}-\frac{2-\left[B^{2}-b\left(2 B^{2}-A B\right) r^{2}\right]}{2\left(1-B^{2} r^{2}\right)}\right| \leq \frac{|b|(A-B) r}{2\left(1-B^{2} r^{2}\right)} .
$$

Proof. If $p(z) \in P(A, B)$, then

$$
\left|p(z)-\frac{1-A B r^{2}}{1-B^{2} r^{2}}\right| \leq \frac{(A-B) r}{1-B^{2} r^{2}} .
$$


The inequality (3.2) was proved by Janowski [7]. Considering Corollary 2.5 and inequality (3.1), then we get

$$
\left|2\left[1+\frac{1}{b}\left(z \frac{f^{\prime}(z)}{f(z)}-1\right)-1\right]-\frac{1-A B r^{2}}{1-B^{2} r^{2}}\right| \leq \frac{(A-B) r}{1-B^{2} r^{2}}
$$

After brief calculations from (3.3), we obtain (3.1).

THEOREM 3.2. The radius of starlikeness for the class $C(A, B, b)$ is

$$
r_{s}=\frac{4}{|b|(A-B)+\sqrt{|b|^{2}(A-B)^{2}+8\left[2 B^{2}+\left(A B-B^{2}\right) \operatorname{Re} b\right]}} .
$$

This radius is sharp, because the extremal function is

$$
f_{*}(z)= \begin{cases}\int_{0}^{z}(1+B \zeta)^{b(A-B) / B} d \zeta, & B \neq 0, \\ \int_{0}^{z} e^{A b \zeta} d \zeta, & B=0 .\end{cases}
$$

Proof. After the brief calculations from inequality (3.1), we get

$$
\operatorname{Re}\left(z \frac{f^{\prime}(z)}{f(z)}\right) \geq \frac{2-|b|(A-B) r-\left[2 B^{2}+\left(A B-B^{2}\right) \operatorname{Re} b\right] r^{2}}{1-B^{2} r^{2}} .
$$

Hence for $r<r_{s}$ the right-hand side of inequality (3.6) is positive. This implies that (3.4) holds.

Also note that inequality (3.6) becomes an equality for the function $f_{*}(z)$. It follows that (3.4) holds.

COROLLARY 3.3. If $A=1, B=-1, b=1$, then $r_{s}=1$. This is the radius of starlikeness of convex functions which is well known (see [1, Volume II, page 88]).

\section{REFERENCES}

[1] O. Altintaş, Ö. Özkan, and H. M. Srivastava, Majorization by starlike functions of complex order, Complex Var. Theory Appl. 46 (2001), no. 3, 207-218.

[2] O. Altintaş and H. M. Srivastava, Some majorization problems associated with $p$-valently starlike and convex functions of complex order, East Asian Math. J. 17 (2001), 175183.

[3] A. Gangadharan, V. Ravichandran, and T. N. Shanmugam, Radii of convexity and strong starlikeness for some classes of analytic functions, J. Math. Anal. Appl. 211 (1997), no. 1, 301-313.

[4] A. W. Goodman, Univalent Functions. Vol. I, Mariner Publishing, Florida, 1983.

[5] _ Univalent Functions. Vol. II, Mariner Publishing, Florida, 1983.

[6] I. S. Jack, Functions starlike and convex of order $\alpha$, J. London Math. Soc. (2) 3 (1971), no. 2, 469-474.

[7] W. Janowski, Some extremal problems for certain families of analytic functions. I, Ann. Polon. Math. 28 (1973), 297-326.

[8] R. J. Libera, Univalent $\alpha$-spiral functions, Canad. J. Math. 19 (1967), 449-456.

[9] R. J. Libera and M. R. Ziegler, Regular functions $f(z)$ for which $z f^{\prime}(z)$ is $\alpha$-spiral, Trans. Amer. Math. Soc. 166 (1972), 361-370. 
[10] M. A. Nasr and M. K. Aouf, On convex functions of complex order, Bulletin of the Faculty of Sciences, University of Mansoura 9 (1982), 566-580.

[11] Y. Polatog̃lu, M. Bolcal, and A. Şen, Two-point distortion theorems for certain families of analytic functions in the unit disc, Int. J. Math. Math. Sci. 2003 (2003), no. 66, 41834193.

[12] M. S. Robertson, Univalent functions $f(z)$ for which $z f^{\prime}(z)$ is spirallike, Michigan Math. J. 16 (1969), 97-101.

[13] E. M. Silvia, A brief overview of subclasses of spiral-like functions, Current Topics in Analytic Function Theory (H. M. Srivastava and S. Owa, eds.), World Scientific, New Jersey, 1992, pp. 328-336.

[14] L. Spacek, Prispevek k teorii funkci prostych, Časopis Pěst. Mat. Fys. 62 (1933), 12-19.

[15] N. Tuneski, On the quotient of the representations of convexity and starlikeness, Math. Nachr. 248/249 (2003), 200-203.

Yaşar Polatog̃lu: Department of Mathematics and Computer Science, Faculty of Science and Letters, Kültür University, Istanbul 34191, Turkey

E-mail address: y. polatoglu@iku.edu.tr

Metin Bolcal: Department of Mathematics and Computer Science, Faculty of Science and Letters, Kültür University, Istanbul 34191, Turkey

E-mail address: m.bolca1@iku.edu.tr

Arzu Şen: Department of Mathematics and Computer Science, Faculty of Science and Letters, Kültür University, Istanbul 34191, Turkey

E-mail address: a.sen@iku.edu.tr 


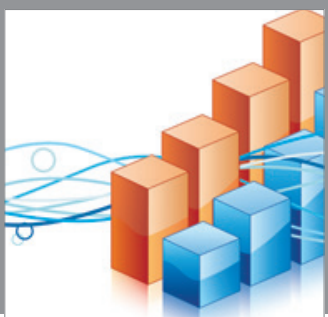

Advances in

Operations Research

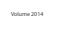

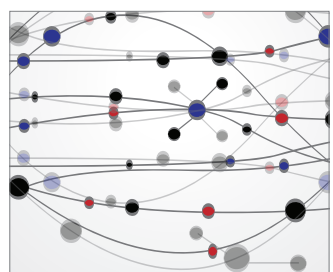

\section{The Scientific} World Journal
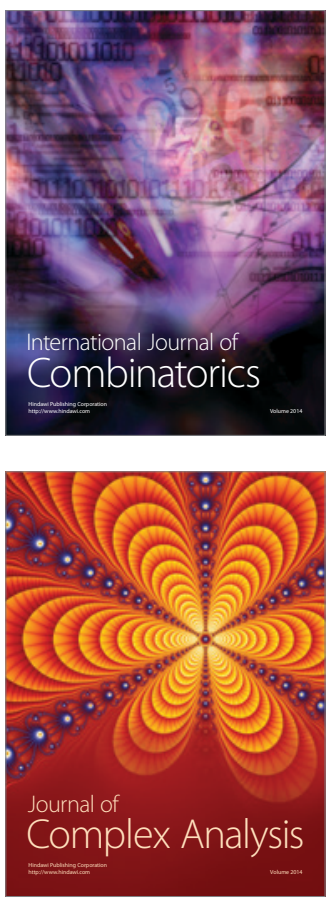

International Journal of

Mathematics and

Mathematical

Sciences
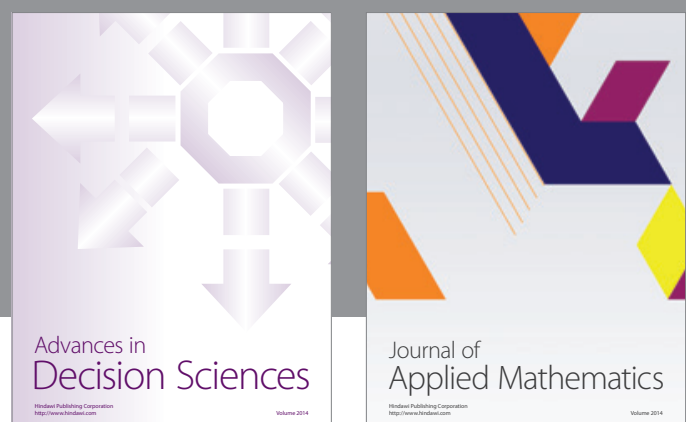

Journal of

Applied Mathematics
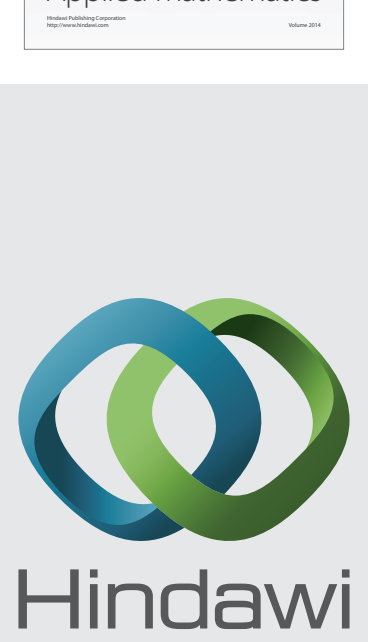

Submit your manuscripts at http://www.hindawi.com
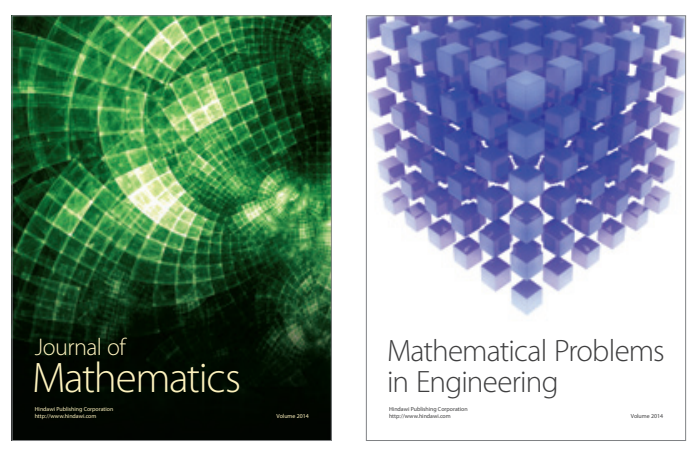

Mathematical Problems in Engineering
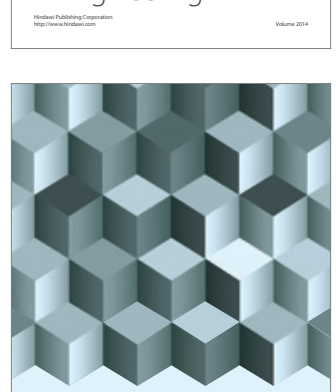

Journal of

Function Spaces
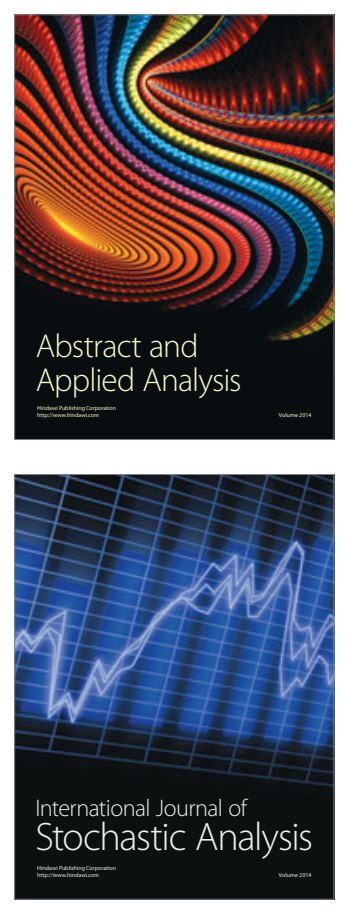

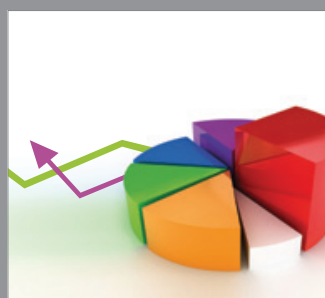

ournal of

Probability and Statistics

Promensencen
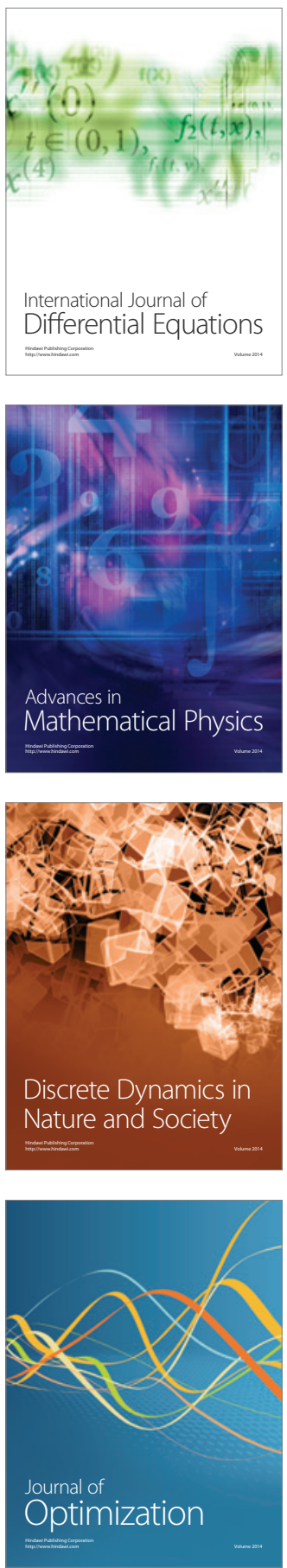